\title{
General decay for a wave equation of Kirchhoff type with a boundary control of memory type
}

\author{
Shun-Tang Wu
}

Correspondence: stwu@ntut.edu.tw General Education Center, National Taipei University of Technology,

Taipei 106, Taiwan

\section{Abstract}

A nonlinear wave equation of Kirchhoff type with memory condition at the boundary in a bounded domain is considered. We establish a general decay result which includes the usual exponential and polynomial decay rates. Furthermore, our results allow certain relaxation functions which are not necessarily of exponential and polynomial decay. This improves earlier results in the literature.

MSC: 35L05; 35L70; 35L75; 74D10.

Keywords: general decay, wave equation, relaxation, memory type, Kirchhoff type, nondissipative

\section{Introduction}

In this article, we study the asymptotic behavior of the energy function related to a nonlinear wave equation of Kirchhoff type subject to memory condition at the boundary as follows:

$$
\begin{aligned}
& u_{t t}-M\left(\|\nabla u\|_{2}^{2}\right) \Delta u+l(t) h(\nabla u)-\Delta u_{t}+a(x) f(u)=0 \text { in } \Omega \times(0, \infty), \\
& u=0 \text { on } \Gamma_{0} \times(0, \infty), \\
& u+\int_{0}^{t} g(t-s)\left(M\left(\|\nabla u(s)\|_{2}^{2}\right) \frac{\partial u}{\partial v}(s)+\frac{\partial u_{t}}{\partial v}(s)\right) d s=0 \text { on } \Gamma_{1} \times(0, \infty), \\
& u(x, 0)=u_{0}(x), u_{t}(x, 0)=u_{1}(x) \text { in } \Omega,
\end{aligned}
$$

where $\Omega$ is a bounded domain with smooth boundary $\partial \Omega=\Gamma_{0} \cup \Gamma_{1}$. The partition $\Gamma_{0}$ and $\Gamma_{1}$ are closed and disjoint, with meas $\left(\Gamma_{0}\right)>0, v$ represents the unit normal vector directed towards the exterior of $\Omega, u$ is the transverse displacement, and $g$ is the relaxation function considered positive and nonincreasing belonging to $W^{1,2}(\Omega)$.

From the physical point of view, we know that the memory effect described in integral equation (1.3) can be caused by the interaction with another viscoelastic element. In fact, the boundary condition (1.3) signifies that $\Omega$ is composed of a material which is clamped in a rigid body in the portion $\Gamma_{0}$ of its boundary and is clamped in a body with viscoelastic properties in the portion of $\Gamma_{1}$.

When $\Gamma_{1}=\varphi$, problem (1.1) has its origin in describing the nonlinear vibrations of an elastic string. More precisely, we have

(C) 2011 Wu; licensee Springer. This is an Open Access article distributed under the terms of the Creative Commons Attribution License (http://creativecommons.org/licenses/by/2.0), which permits unrestricted use, distribution, and reproduction in any medium, provided the original work is properly cited. 


$$
\rho h \frac{\partial^{2} u}{\partial t^{2}}=\left\{p_{0}+\frac{E h}{2 L} \int_{0}^{L}\left(\frac{\partial u}{\partial x}\right)^{2} d x\right\} \frac{\partial^{2} u}{\partial x^{2}}+f
$$

for $0<x<L, t \geq 0$; where $u$ is the lateral deflection, $x$ the space coordinate, $t$ the time, $E$ the Young modulus, $\rho$ the mass density, $h$ the cross section area, $L$ the length, $p_{0}$ the initial axial tension and $f$ the external force. Kirchhoff [1] was the first one who introduced (1.5) to study the oscillations of stretched strings and plates, so that (1.5) is called the wave equation of Kirchhoff type after him. In this direction, problem (1.1) with $\partial \Omega=\Gamma_{0}$ and $l(t)=0$ has been investigated by many authors in recent years, and many results concerning existence, nonexistence and asymptotic behavior have been established, see [2-13].

On the other hand, regarding the viscoelastic wave equations with memory term acting in the boundary or in the domain, there are numerous results related to asymptotic behavior of solutions. For example, in the case where $M(s)=1$, Cavalcanti et al. [14] investigated the existence and uniform decay of strong solutions of wave equation (1.1) with a nonlinear boundary damping of memory type and a nonlinear boundary source when $l(t)=0$. Cavalcanti and Guesmia [15] considered the following system:

$$
\begin{aligned}
& u_{t t}-\Delta u+F(x, t, u, \nabla u)=0 \text { in } \Omega \times(0, \infty), \\
& u=0 \text { on } \Gamma_{0} \times(0, \infty), \\
& u+\int_{0}^{t} g(t-s) \frac{\partial u}{\partial v}(s) d s=0 \text { on } \Gamma_{1} \times(0, \infty), \\
& u(x, 0)=u_{0}(x), u_{t}(x, 0)=u_{1}(x), \text { in } \Omega,
\end{aligned}
$$

where $\Omega$ is a bounded domain with smooth boundary $\partial \Omega=\Gamma_{0} \cup \Gamma_{1}$. They obtained the general decay result which depends on the relaxation function $g$. In particular, if the relaxation function $g$ decays exponentially (or polynomially), then the solution also decays exponentially (or polynomially) and with the same decay rate. Moreover, when $u_{0}=0$ on $\Gamma_{1}$, they obtained exponential or polynomial decay of solutions, even if the relaxation function $g$ does not converge to 0 at $\infty$. Later, Messaoudi and Soufyane [16] generalized this result to the case of a system of Timoshenko type. They established general decay rate results, from which the usual exponential and polynomial decay rates are only special cases. Recently, Messaoudi and Soufyane [17] studied the following problem:

$$
u_{t t}-\Delta u+f(u)=0
$$

in a bounded domain with boundary conditions (1.7)-(1.9). They improved the results of [15] by applying the multiplier techniques. Indeed, they obtained not only a general decay result, but their works also allowed certain relaxation functions which are not necessarily of exponential or polynomial decay. For other related works, we refer the reader to [18-20] and references therein.

Conversely, in the case where $M$ is not a constant function, Santos [21] considered

$$
\begin{aligned}
u_{t t}-\mu(t) u_{x x} & =0, \quad(x, t) \in(0,1) \times R^{+}, \\
u(0, t) & =0, \quad u(1, t)=-\int_{0}^{t} g(t-s) \mu(s) u_{x}(1, s) d s, \quad \forall t>0, \\
u(0) & =u_{0}, \quad u_{t}(0)=u_{1}, \quad x \in(0,1),
\end{aligned}
$$


where $\mu(t)$ is a nonincreasing function satisfying $\mu(t) \geq \mu_{0}>0$. By denoting $k$ the resolvent kernel of $g$, he showed that the solution decays exponentially (or polynomially) to zero provided $k$ decays exponentially (or polynomially) to zero. Later on, Santos et al. [22] generalized this result to a nonlinear $n$-dimensional equation of Kirchhoff type of the form

$$
u_{t t}-M\left(\|\nabla u\|_{2}^{2}\right) \Delta u-\Delta u_{t}+f(u)=0
$$

in a bounded domain with boundary conditions (1.2)-(1.3). In that article, they proved that the energy decays with the same rate of decay of the relaxation function. This latter result improved an earlier one by Park et al. [23], where the authors considered (1.10) in a bounded domain with nonlinear boundary damping and memory term and $M(s)=1+s$ and $f=0$.

We note that stability of problems with the nonlinear term $h(\nabla u)$ requires a careful treatment because we do not have any information about the influence of the integral $\int_{\Omega} h(\nabla u) u_{t} d x$ about the sign of the derivative $E^{\prime}(t)$. Although the subject is important, there are few mathematical results in the presence of the nonlinearity given by $h(\nabla u)$, see [24-26]. In light of this and previous articles [17,22], it is interesting to investigate whether we still have the similar general decay result as in [17] for nondissipative distributed system (1.1) with the memory-type damping acting on a part of the boundary. Hence, the main purpose of this article is to answer the above question for system (1.1)-(1.4). Consequently, by following the arguments close to the one in [17] with necessary modification required the nature of our problem, we establish a general decay result which includes the usual exponential and polynomial decay rates. Furthermore, our results allow a larger class of relax functions which are not necessarily of exponential and polynomial decay. Therefore, this improves earlier results in the literature $[22,27]$.

In order to obtain our results, we consider system (1.1)-(1.4), under some assumptions on $a(x), l(t), M$ and $f$. Precisely, we state the general assumptions:

(A1) $a(x): \Omega \rightarrow R^{+}$is a function.

(A2) $f \in C^{1}(R)$ is a function and satisfies

$$
u f(u) \geq \beta F(u) \geq 0 \quad \text { for } \quad \beta>2,
$$

where $F(u)=\int_{0}^{u} f(s) d s$ with

$$
F(u) \leq d|u|^{p} \quad \text { for all } u \in R,
$$

$d>0$ and $1 \leq p \leq \frac{n}{n-2}$.

(A3) $M$ is a $C^{1}$ function on $[0, \infty)$ satisfying

$$
M(\lambda) \geq m_{0}>0 \text { and } M(\lambda) \lambda \geq \widehat{M}(\lambda) \text { for all } \lambda \geq 0,
$$

Where $\widehat{M}(\lambda)=\int_{0}^{\lambda} M(s) d s$.

(A4) $h: R^{n} \rightarrow R$ is a $C^{1}$ function such that $\nabla h$ is bounded and there exists $\beta_{1}>0$ such that

$$
|h(\xi)| \leq \beta_{1}|\xi| \quad \text { for all } \xi \in R^{n},
$$


and $l(t)$ is a positive and nonincreasing function.

The remainder of this article is organized as follows. In Section 2, we introduce some notations, present Lemma 2.1 to describe more general relations between the relaxation function $g$ and the corresponding resolvent kernel $k$ and state the existence result to system (1.1)-(1.4). In Section 3, we give the proof of our main result Theorem 3.5.

\section{Preliminaries}

In this section, we introduce some notations and establish the existence of solutions of the problem (1.1)-(1.4). In what follows, let $\|\cdot\|_{p}$ denote the usual $L^{p}(\Omega)$ norm $\|\cdot\|_{L^{p}(\Omega)}$, for $1 \leq p \leq \infty$. We define the convolution product operator by

$$
(g * u)(t)=\int_{0}^{t} g(t-s) u(s) d s,
$$

and set

$$
\begin{aligned}
& (g \circ \phi)(t)=\int_{0}^{t} g(t-s)\|\phi(t)-\phi(s)\|_{2}^{2} d s, \\
& (g \diamond \phi)(t)=\int_{0}^{t} g(t-s)(\phi(t)-\phi(s)) d s .
\end{aligned}
$$

Using Hölder's inequality, we observe that

$$
|g \diamond \phi(t)|^{2} \leq \int_{0}^{t}|g(s)| d s(|g| \circ \phi)(t) .
$$

Next, we shall use Equation 1.3 to estimate the boundary term $M\left(\|\nabla u(s)\|_{2}^{2}\right) \frac{\partial u}{\partial v}+\frac{\partial u_{t}}{\partial v}$. Differentiating (1.3), we obtain

$$
\begin{aligned}
M & \left(\|\nabla u(t)\|_{2}^{2}\right) \frac{\partial u}{\partial v}(t)+\frac{\partial u_{t}}{\partial v}(t)+\frac{1}{g(0)} \int_{0}^{t} g^{\prime}(t-s)\left(M\left(\|\nabla u(s)\|_{2}^{2}\right) \frac{\partial u}{\partial v}(s)+\frac{\partial u_{t}}{\partial v}(s)\right) d s \\
& =-\frac{1}{g(0)} u_{t} .
\end{aligned}
$$

Assume the function $k$ is the resolvent kernel of the relaxation function $g$, then

$$
k+\frac{1}{g(0)} k * g^{\prime}=-\frac{1}{g(0)} g^{\prime} .
$$

Applying Volterra's inverse operator yields

$$
M\left(\|\nabla u(t)\|_{2}^{2}\right) \frac{\partial u}{\partial v}(t)+\frac{\partial u_{t}}{\partial v}(t)=-\frac{1}{g(0)}\left(u_{t}+k * u_{t}\right),
$$

which implies that

$$
\begin{aligned}
M & \left(\|\nabla u(t)\|_{2}^{2}\right) \frac{\partial u}{\partial v}(t)+\frac{\partial u_{t}}{\partial v}(t) \\
& =-\tau\left\{u_{t}+k(0) u-k(t) u_{0}+k^{\prime} * u\right\} \text { on } \Gamma_{1} \times(0, \infty),
\end{aligned}
$$

where $\tau=\frac{1}{g(0)}$. Reciprocally, taking $u_{0}=0$ on $\Gamma_{1}$, identity (2.5) implies (1.3). As we are interested in relaxation functions of more general decay and the resolvent $k$ 
appeared in Equation 2.5, we want to know if the resolvent $k$ has the same property with the relaxation function $g$ involved in (1.3). The following lemma answers this question. Let $h$ be a relaxation function and $k$ its resolvent kernel, that is

$$
k(t)=h(t)+(k * h)(t) .
$$

Lemma 2.1. $[15,17,22]$ If $h:[0, \infty) \rightarrow R^{+}$is continuous, then $k$ is also a positive continuous function. Moreover,

(1) If there exists a positive constant $c_{0}$ such that

$$
h(t) \leq c_{0} e^{-\int_{0}^{t} \gamma(s) d s},
$$

where $\gamma:[0, \infty) \rightarrow R^{+}$, is a nonincreasing function satisfying, for some positive constant $\varepsilon<1$,

$$
c_{1}=\int_{0}^{\infty} e^{-\int_{0}^{t}(1-\epsilon) \gamma(s) d s} d t<\frac{1}{c_{0}} .
$$

Then, $k$ satisfies

$$
k(t) \leq \frac{c_{0}}{1-c_{0} c_{1}} e^{-\int_{0}^{t} \varepsilon \gamma(s) d s} .
$$

(2) Suppose that

$$
h(t) \leq \frac{c_{0}}{(1+t)^{p}}
$$

for $c_{0}<p-1$. Then, there exists a positive constant $\varepsilon<1$ such that

$$
k(t) \leq \frac{\beta}{(1+t)^{\varepsilon p}},
$$

where $\beta>0$ is a constant.

Based on this lemma, we will use (2.5) instead of (1.3), i.e., we can consider system (1.1)-(1.4) as follows:

$$
\begin{gathered}
u_{t t}-M\left(\|\nabla u\|_{2}^{2}\right) \Delta u+l(t) h(\nabla u)-\Delta u_{t}+a(x) f(u)=0 \text { in } \Omega \times(0, \infty) \\
u=0 \text { on } \Gamma_{0} \times(0, \infty) \\
M\left(\|\nabla u(t)\|_{2}^{2}\right) \frac{\partial u}{\partial v}(t)+\frac{\partial u_{t}}{\partial v}(t)=-\tau\left\{u_{t}+k(0) u-k(t) u_{0}+k^{\prime} * u\right\} \text { on } \Gamma_{1} \times(0, \infty), \\
u(x, 0)=u_{0}(x), u_{t}(x, 0)=u_{1}(x) \text { in } \Omega .
\end{gathered}
$$

We notice that, due to the condition (1.2), the solution of system (1.1)-(1.4) must belong to the following space:

$$
V=\left\{v \in H^{1}(\Omega) ; \quad v=0 \text { on } \Gamma_{0}\right\},
$$

which endowed with the norm $\|\nabla \cdot\|_{2}$ is a Hilbert space. Now, we are ready to give the well-posedness of system (1.1)-(1.4).

Theorem 2.2. Let $k \in W^{2,1}\left(R^{+}\right) \cap W^{1, \infty}\left(R^{+}\right),\left(u_{0}, u_{1}\right) \in\left(H^{2}(\Omega) \cap V\right)^{2}$ and satisfy the compatibility condition

$$
M\left(\left\|\nabla u_{0}\right\|_{2}^{2}\right) \frac{\partial u_{0}}{\partial v}+\frac{\partial u_{1}}{\partial v}+\tau u_{1}=0 \text { on } \Gamma_{1} .
$$


Assume further that (A1)-(A4) hold. Then, there exists a unique solution u of system (1.1)-(1.4) such that

$$
\begin{aligned}
u & \in L^{\infty}\left(0, \infty ; H^{2}(\Omega) \cap V\right), \quad u_{t} \in L^{\infty}(0, \infty ; V), \\
u_{t t} & \in L^{\infty}\left(0, \infty ; L^{2}(\Omega)\right) .
\end{aligned}
$$

Proof. Using the Galerkin method and procedures similar to that of $[22,28]$, we can obtain the result.

\section{Decay of solutions}

In this section, we study the asymptotic behavior of the solutions of system (1.1)-(1.4) when the resolvent kernel $k$ satisfies

$$
k(0)>0, \quad k(t) \geq 0, \quad k^{\prime}(t) \leq 0, \quad k^{\prime \prime}(t) \geq-\gamma(t) k^{\prime}(t),
$$

where $\gamma:[0, \infty) \rightarrow R^{+}$is a function satisfying the following condition:

$$
\gamma(t)>0, \quad \gamma^{\prime}(t) \leq 0 \quad \text { and } \quad \int_{0}^{\infty} \gamma(s) d s=\infty .
$$

To get our result, we further assume that

$$
0<l(t) \leq \gamma(t) \text { for all } t \geq 0 .
$$

Let $x_{0}$ be a fixed point in $R^{n}$. Set

$$
m=m(x)=x-x_{0}, \quad R\left(x_{0}\right)=\max \left\{\|m(x)\|_{2} ; x \in \bar{\Omega}\right\}
$$

and partition the boundary $\partial \Omega$ into two sets

$$
\Gamma_{0}=\{x \in \partial \Omega ; m(x) \cdot v \leq 0\}, \quad \Gamma_{1}=\{x \in \partial \Omega ; m(x) \cdot v>0\} .
$$

Define the first-order energy function of system (1.1)-(1.4) by

$$
\begin{aligned}
E(t)= & \frac{1}{2}\left(\left\|u_{t}\right\|_{2}^{2}+\widehat{M}\left(\|\nabla u\|_{2}^{2}\right)\right)+\int_{\Omega} a(x) F(u) d x \\
& +\frac{\tau}{2} k(t) \int_{\Gamma_{1}}|u|^{2} d \Gamma-\frac{\tau}{2} \int_{\Gamma_{1}} k^{\prime} \circ u d \Gamma .
\end{aligned}
$$

The following lemma is associated with the property of the convolution operator, which is used to estimate the energy identity.

Lemma 3.1. If $g, \varphi \in C^{1}\left(R^{+}\right)$, then

$$
\begin{aligned}
(g * \phi) \phi_{t}= & -\frac{1}{2} g(t)|\phi(t)|^{2}+\frac{1}{2} g^{\prime} \circ \phi \\
& -\frac{1}{2} \frac{d}{d t}\left(g \circ \phi-\left(\int_{0}^{t} g(s) d s\right)|\phi(t)|^{2}\right) .
\end{aligned}
$$

Proof. Our conclusion is followed by differentiating the term $g \circ \varphi$.

Lemma 3.2. Under the assumptions of (A1)-(A4), the energy function $E(t)$ satisfies

$$
\begin{aligned}
\frac{d}{d t} E(t) \leq & \frac{-\tau}{2} \int_{\Gamma_{1}}\left|u_{t}\right|^{2} d \Gamma+\frac{\tau}{2} k^{2}(t) \int_{\Gamma_{1}}\left|u_{0}\right|^{2} d \Gamma+\frac{\tau}{2} k^{\prime}(t) \int_{\Gamma_{1}}|u|^{2} d \Gamma \\
& -\frac{\tau}{2} \int_{\Gamma_{1}} k^{\prime \prime} \circ u d \Gamma-\int_{\Omega}\left|\nabla u_{t}\right|^{2} d x-\int_{\Omega} l(t) h(\nabla u) u_{t} d x .
\end{aligned}
$$


Proof. Multiplying Equation 1.1 by $u_{t}$, and integrating by parts over $\Omega$, we get

$$
\begin{aligned}
\frac{d}{d t} & {\left[\frac{1}{2}\left(\left\|u_{t}\right\|_{2}^{2}+\widehat{M}\left(\|\nabla u\|_{2}^{2}\right)\right)+\int_{\Omega} a(x) F(u) d x\right] } \\
& =\int_{\Gamma_{1}}\left(M\left(\|\nabla u\|_{2}^{2}\right) \frac{\partial u}{\partial v}+\frac{\partial u_{t}}{\partial v}\right) u_{t} d \Gamma-\int_{\Omega}\left|\nabla u_{t}\right|^{2} d x-\int_{\Omega} \Delta l(t) h(\nabla u) u_{t} d x .
\end{aligned}
$$

Exploiting (2.5), (3.6) and the definition of $E(t)$ by (3.5), we have

$$
\begin{aligned}
\frac{d}{d t} E(t) \leq & -\tau \int_{\Gamma_{1}}\left|u_{t}\right|^{2} d \Gamma+\tau \int_{\Gamma_{1}} k(t) u_{0} u_{t} d \Gamma+\frac{\tau}{2} k^{\prime}(t) \int_{\Gamma_{1}}|u|^{2} d \Gamma \\
& -\frac{\tau}{2} \int_{\Gamma_{1}} k^{\prime \prime} \circ u d \Gamma-\int_{\Omega}\left|\nabla u_{t}\right|^{2} d x-\int_{\Omega} l(t) h(\nabla u) u_{t} d x
\end{aligned}
$$

Then, using Hölder's inequality and Young's inequality, the inequality (3.7) is obtained.

Next, we construct a Lyapunov functional which is equivalent to $E(t)$. To do so, for $N>0$ large enough, let

$$
L(t)=N E(t)+\psi(t)
$$

where

$$
\psi(t)=\int_{\Omega}\left(m \cdot \nabla u(t)+\left(\frac{n}{2}-\theta\right) u\right) u_{t} d x
$$

for $0<\theta<1$.

For the purpose of achieving our main result, we need the following lemmas.

Lemma 3.3. There exist two positive constants $\alpha_{1}$ and $\alpha_{2}$ such that the relation

$$
\alpha_{1} E(t) \leq L(t) \leq \alpha_{2} E(t)
$$

holds for all $t \geq 0$.

Proof. From (3.9) and using Young's inequality, we get

$$
\begin{aligned}
|\psi(t)| & \leq\left(R\left(x^{0}\right)+B_{1}\left(\frac{n}{2}-\theta\right)\right)\left\|u_{t}\right\|_{2}\|\nabla u\|_{2} \\
& \leq \frac{2}{\sqrt{m_{0}}}\left(R\left(x^{0}\right)+B_{1}\left(\frac{n}{2}-\theta\right)\right) E(t)
\end{aligned}
$$

where we have used the fact that $\left\|u_{t}\right\|_{2}^{2} \leq 2 E(t)$ by (3.5) and

$$
\|\nabla u\|_{2}^{2} \leq \frac{2}{m_{0}} E(t)
$$

due to $\hat{M}(\lambda) \geq m_{0} \lambda>0$ by (A3) and (3.5). Here $B_{1}>0$ is the smallest constant such that

$$
\|u\|_{2} \leq B_{1}\|\nabla u\|_{2}, \quad \forall u \in V .
$$

Thus, from (3.8), we deduce that

$$
|L(t)-N E(t)|=|\psi(t)| \leq \frac{2}{\sqrt{m_{0}}}\left(R\left(x^{0}\right)+B_{1}\left(\frac{n}{2}-\theta\right)\right) E(t) .
$$


Hence, selecting

$$
N>\frac{2}{\sqrt{m_{0}}}\left(R\left(x^{0}\right)+B_{1}\left(\frac{n}{2}-\theta\right)\right),
$$

there exist two positive constants $\alpha_{1}$ and $\alpha_{2}$ such that the relation

$$
\alpha_{1} E(t) \leq L(t) \leq \alpha_{2} E(t)
$$

holds. $\square$

Lemma 3.4. Let (A1)-(A4) and (3.1)-(3.3) hold, with $\beta_{1}$ (given by (A4)) small enough and

$$
\lim _{t \rightarrow \infty} k(t)=0 .
$$

Then, for some $t_{0}$ large enough, the functional $L(t)$ verifies, along the solution $u$ of (1.1)-(1.4),

$$
\begin{aligned}
L^{\prime}(t) \leq & -\frac{\alpha}{2} E(t)+c_{4} k^{2}(t) \int_{\Gamma_{1}}\left|u_{0}\right|^{2} d \Gamma-c_{5} \int_{\Gamma_{1}} k^{\prime} \circ u d \Gamma \\
& +\int_{\Omega}\left[\left(n+\alpha-\left(\frac{n}{2}-\theta\right) \beta\right) a(x)+m \cdot \nabla a\right] F(u) d x
\end{aligned}
$$

for all $t \geq t_{0}$, where $\alpha=\min \{2 \theta, 1-\theta\}$ and $c_{i}$ are positive constants given in the proof, $i=4,5$.

Proof. First, we are going to estimate the derivative of $\psi(t)$. From (3.9) and using Equation 1.1, we have

$$
\begin{aligned}
\frac{d}{d t} \psi(t)= & \frac{1}{2} \int_{\Gamma_{1}}(m \cdot v)\left|u_{t}\right|^{2} d \Gamma-\theta \int_{\Omega}\left|u_{t}\right|^{2} d x \\
& +\int_{\Omega}\left(m \cdot \nabla u(t)+\left(\frac{n}{2}-\theta\right) u\right) M\left(\|\nabla u\|_{2}^{2}\right) \Delta u d x \\
& +\int_{\Omega}\left(m \cdot \nabla u(t)+\left(\frac{n}{2}-\theta\right) u\right) \Delta u_{t} d x \\
& -\int_{\Omega} l(t) h(\nabla u)\left(m \cdot \nabla u(t)+\left(\frac{n}{2}-\theta\right) u\right) d x \\
& -\int_{\Omega}\left(m \cdot \nabla u(t)+\left(\frac{n}{2}-\theta\right) u\right) a(x) f(u) d x
\end{aligned}
$$

Performing integration by parts and using Young's inequality, we obtain

$$
\begin{aligned}
\frac{d}{d t} \psi(t) \leq & \frac{1}{2} \int_{\Gamma_{1}}(m \cdot v)\left|u_{t}\right|^{2} d \Gamma-\theta \int_{\Omega}\left|u_{t}\right|^{2} d x \\
& +\int_{\Gamma_{1}}\left(M\left(|| \nabla u \|_{2}^{2}\right) \frac{\partial u}{\partial v}+\frac{\partial u_{t}}{\partial v}\right)\left(m \cdot \nabla u(t)+\left(\frac{n}{2}-\theta\right) u\right) d \Gamma \\
& -\frac{M\left(|| \nabla u \|_{2}^{2}\right)}{2} \int_{\Gamma_{1}}(m \cdot v)|\nabla u|^{2} d \Gamma-(1-\theta) M\left(\|\nabla u\|_{2}^{2}\right)\|\nabla u\|_{2}^{2} \\
& +\varepsilon \mathcal{c}_{0} M\left(\|\nabla u\|_{2}^{2}\right)\|\nabla u\|_{2}^{2}+C_{\varepsilon} \int_{\Omega}\left|\nabla u_{t}\right|^{2} d x \\
& -\int_{\Omega} l(t) h(\nabla u)\left(m \cdot \nabla u(t)+\left(\frac{n}{2}-\theta\right) u\right) d x \\
& -\int_{\Omega}\left(m \cdot \nabla u(t)+\left(\frac{n}{2}-\theta\right) u\right) a(x) f(u) d x
\end{aligned}
$$


where $\varepsilon>0, c_{\varepsilon}$ and $c_{0}$ are some positive constants. In the following, we will estimate the last two terms on the right-hand side of (3.15). It follows from (1.13), Hölder's inequality, (3.11), (3.3) and (3.10) that

$$
\begin{aligned}
& \int_{\Omega} l(t) h(\nabla u)\left(m \cdot \nabla u(t)+\left(\frac{n}{2}-\theta\right) u\right) d x \\
& \quad \leq \gamma(0) \beta_{1}\left(R\left(x^{0}\right)+B_{1}\left(\frac{n}{2}-\theta\right)\right)\|\nabla u\|_{2}^{2} \\
& \quad \leq \frac{2 \gamma(0) \beta_{1} c_{1}}{m_{0}} E(t),
\end{aligned}
$$

where $c_{1}=R\left(x^{0}\right)+B_{1}\left(\frac{n}{2}-\theta\right)$. Taking (1.11) and (3.4) into account, we have

$$
\begin{aligned}
& -\int_{\Omega}\left(m \cdot \nabla u(t)+\left(\frac{n}{2}-\theta\right) u\right) a(x) f(u) d x \\
& \quad=-\int_{\Omega} a(x) m \cdot \nabla F(u) d x-\left(\frac{n}{2}-\theta\right) \int_{\Omega} a(x) u f(u) d x \\
& \quad \leq \int_{\Omega}(n a(x)+m \cdot \nabla a) F(u) d x-\int_{\Gamma_{1}} a(x)(m \cdot v) F(u) d \Gamma \\
& -\left(\frac{n}{2}-\theta\right) \beta \int_{\Omega} a(x) F(u) d x \\
& \quad \leq \int_{\Omega}\left[\left(n-\left(\frac{n}{2}-\theta\right) \beta\right) a(x)+m \cdot \nabla a\right] F(u) d x .
\end{aligned}
$$

A substitution of (3.16)-(3.17) into (3.15), we obtain

$$
\begin{aligned}
\frac{d}{d t} \psi(t) \leq & \frac{1}{2} \int_{\Gamma_{1}}(m \cdot v)\left|u_{t}\right|^{2} d \Gamma-\theta\left\|u_{t}\right\|_{2}^{2}-\left(1-\theta-\varepsilon c_{0}\right) M\left(\|\nabla u\|_{2}^{2}\right)\|\nabla u\|_{2}^{2} \\
& +\int_{\Gamma_{1}}\left(M\left(\|\nabla u\|_{2}^{2}\right) \frac{\partial u}{\partial v}+\frac{\partial u_{t}}{\partial v}\right)\left(m \cdot \nabla u(t)+\left(\frac{n}{2}-\theta\right) u\right) d \Gamma \\
& +C_{\varepsilon} \int_{\Omega}\left|\nabla u_{t}\right|^{2} d x-\frac{M\left(\|\nabla u\|_{2}^{2}\right)}{2} \int_{\Gamma_{1}}(m \cdot v)|\nabla u|^{2} d \Gamma+\frac{2 \gamma(0) \beta_{1} c_{1}}{m_{0}} E(t) \\
& +\int_{\Omega}\left[\left(n-\left(\frac{n}{2}-\theta\right) \beta\right) a(x)+m \cdot \nabla a\right] F(u) d x .
\end{aligned}
$$

Now, we analyze the boundary term on the right-hand side of (3.18). Applying Young's inequality and $M(\lambda) \geq m_{0}>0$ by (1.12), we have, for $\varepsilon_{1}>0$,

$$
\begin{aligned}
& \int_{\Gamma_{1}}\left(M\left(\|\nabla u\|_{2}^{2}\right) \frac{\partial u}{\partial v}+\frac{\partial u_{t}}{\partial v}\right)\left(m \cdot \nabla u(t)+\left(\frac{n}{2}-\theta\right) u\right) d \Gamma \\
& \quad \leq \varepsilon_{1} \int_{\Gamma_{1}}\left[|m \cdot \nabla u(t)|^{2}+\left(\frac{n}{2}-\theta\right)^{2}|u|^{2}\right] d \Gamma+C_{\varepsilon_{1}} \int_{\Gamma_{1}}\left|M\left(\|\nabla u\|_{2}^{2}\right) \frac{\partial u}{\partial v}+\frac{\partial u_{t}}{\partial v}\right|^{2} d \Gamma \\
& \quad \leq \varepsilon_{1} \int_{\Gamma_{1}}(m \cdot v)|\nabla u|^{2} d \Gamma+\left(\frac{n}{2}-\theta\right)^{2} B_{*} \varepsilon_{1}\|\nabla u\|_{2}^{2}+C_{\varepsilon_{1}} \int_{\Gamma_{1}}\left|M\left(\|\nabla u\|_{2}^{2}\right) \frac{\partial u}{\partial v}+\frac{\partial u_{t}}{\partial v}\right|^{2} d \Gamma \\
& \quad \leq \varepsilon_{1} \int_{\Gamma_{1}}(m \cdot v)|\nabla u|^{2} d \Gamma+\left(\frac{n}{2}-\theta\right)^{2} \frac{B_{*} \varepsilon_{1}}{m_{0}} M\left(\|\nabla u\|_{2}^{2}\right)\|\nabla u\|_{2}^{2} \\
& \quad+C_{\varepsilon_{1}} \int_{\Gamma_{1}}\left|M\left(\|\nabla u\|_{2}^{2}\right) \frac{\partial u}{\partial v}+\frac{\partial u_{t}}{\partial v}\right|^{2} d \Gamma,
\end{aligned}
$$

where $C_{\varepsilon_{1}}$ is a positive constant and $B *>0$ is the constant such that

$$
\int_{\Gamma_{1}}|u|^{2} d \Gamma \leq B_{*}\|\nabla u\|_{2}^{2}, \quad \forall u \in V
$$


Thus, (3.18) becomes

$$
\begin{aligned}
\frac{d}{d t} \psi(t) \leq & \frac{1}{2} \int_{\Gamma_{1}}(m \cdot v)\left|u_{t}\right|^{2} d \Gamma-\theta\left\|u_{t}\right\|_{2}^{2} \\
& -\left(1-\theta-\varepsilon c_{0}-\left(\frac{n}{2}-\theta\right)^{2} \frac{B_{*} \varepsilon_{1}}{m_{0}}\right) M\left(\|\nabla u\|_{2}^{2}\right)\|\nabla u\|_{2}^{2} \\
& -\left(\frac{M\left(\|\nabla u\|_{2}^{2}\right)}{2}-\varepsilon_{1}\right) \int_{\Gamma_{1}}(m \cdot v)|\nabla u|^{2} d \Gamma+C_{\varepsilon} \int_{\Omega}\left|\nabla u_{t}\right|^{2} d x \\
& +C_{\varepsilon} \int_{\Gamma_{1}}\left|M\left(\|\nabla u\|_{2}^{2}\right) \frac{\partial u}{\partial v}+\frac{\partial u_{t}}{\partial v}\right|^{2} d \Gamma+\frac{2 \gamma(0) \beta_{1} c_{1}}{m_{0}} E(t) \\
& +\int_{\Omega}\left[\left(n-\left(\frac{n}{2}-\theta\right) \beta\right) a(x)+m \cdot \nabla a\right] F(u) d x .
\end{aligned}
$$

By rewriting the boundary condition (2.5) as

$$
M\left(\|\nabla u\|_{2}^{2}\right) \frac{\partial u}{\partial v}+\frac{\partial u_{t}}{\partial v}=-\tau\left\{u_{t}+k(t) u(t)-k(t) u 0-k^{\prime} \diamond u\right\},
$$

and, then, combining (3.7) and (3.20), we deduce that

$$
\begin{aligned}
L^{\prime}(t)= & N E^{\prime}(t)+\psi^{\prime}(t) \\
\leq & -\left(\frac{N \tau}{2}-\frac{(m \cdot v)}{2}-8 \tau^{2} c_{\varepsilon_{1}}\right) \int_{\Gamma_{1}}\left|u_{t}\right|^{2} d \Gamma-\left(N-c_{\varepsilon}\right)\left\|\nabla u_{t}\right\|_{2}^{2} \\
& -\theta\left\|u_{t}\right\|_{2}^{2}-\left(1-\theta-\varepsilon c_{0}-\left(\frac{n}{2}-\theta\right)^{2} \frac{B_{*} \varepsilon_{1}}{m_{0}}\right) M\left(\|\nabla u\|_{2}^{2}\right)\|\nabla u\|_{2}^{2} \\
& +8 \tau^{2} c_{\varepsilon_{1}} k^{2}(t) \int_{\Gamma_{1}}|u|^{2} d \Gamma+\left(\frac{N \tau}{2}+8 \tau^{2} c_{\varepsilon_{1}}\right) k^{2}(t) \int_{\Gamma_{1}}\left|u_{0}\right|^{2} d \Gamma \\
& -\frac{N \tau}{2} \int_{\Gamma_{1}} k^{\prime \prime} \circ u d \Gamma-\left(\frac{M\left(\|\nabla u\|_{2}^{2}\right)}{2}-\varepsilon_{1}\right) \int_{\Gamma_{1}}(m \cdot v)|\nabla u|^{2} d \Gamma \\
& +8 \tau^{2} c_{\varepsilon_{1}} \int_{\Gamma_{1}}\left|k^{\prime} \diamond u\right|^{2} d \Gamma+\frac{2 \gamma(0) \beta_{1} c_{1}}{m_{0}} E(t)-N l(t) \int_{\Omega} h(\nabla u) u_{t} d x \\
& +\int_{\Omega}\left[\left(n-\left(\frac{n}{2}-\theta\right) \beta\right) a(x)+m \cdot \nabla a\right] F(u) d x .
\end{aligned}
$$

Similarly as in deriving (3.16), we note that

$$
\begin{aligned}
l(t) \int_{\Omega} h(\nabla u) u_{t} d x & \leq \gamma(t) \beta_{1}\left(\frac{1}{2}\left\|u_{t}\right\|_{2}^{2}+\frac{1}{2}\|\nabla u\|_{2}^{2}\right) \\
& \leq \gamma(t) \beta_{1} c_{3} E(t) \leq \gamma(0) \beta_{1} c_{3} E(t),
\end{aligned}
$$

where $c_{3}=1+\frac{1}{m_{0}}$. This implies that

$$
\begin{aligned}
L^{\prime}(t) \leq & -\left(\frac{N \tau}{2}-\frac{(m \cdot v)}{2}-8 \tau^{2} c_{\varepsilon_{1}}\right) \int_{\Gamma_{1}}\left|u_{t}\right|^{2} d \Gamma \\
& -\theta\left\|u_{t}\right\|_{2}^{2}-\left(N-c_{\varepsilon}\right)|| \nabla u_{t} \|_{2}^{2} \\
& -\left(1-\theta-\varepsilon c_{0}-\left(\frac{n}{2}-\theta\right)^{2} \frac{B_{*} \varepsilon_{1}}{m_{0}}\right) M\left(\|\nabla u\|_{2}^{2}\right)\|\nabla u\|_{2}^{2} \\
& +8 \tau^{2} c_{\varepsilon_{1}} k^{2}(t) \int_{\Gamma_{1}}|u|^{2} d \Gamma+\left(\frac{N \tau}{2}+8 \tau^{2} c_{\varepsilon_{1}}\right) k^{2}(t) \int_{\Gamma_{1}}\left|u_{0}\right|^{2} d \Gamma \\
& -\frac{N \tau}{2} \int_{\Gamma_{1}} k^{\prime \prime} \circ u d \Gamma-\left(\frac{M\left(\|\nabla u\|_{2}^{2}\right)}{2}-\varepsilon_{1}\right) \int_{\Gamma_{1}}(m \cdot v)|\nabla u|^{2} d \Gamma \\
& +8 \tau^{2} c_{\varepsilon_{1}} \int_{\Gamma_{1}}\left|k^{\prime} \diamond u\right|^{2} d \Gamma+\beta_{1} \gamma(0)\left(N c_{3}+\frac{2 c_{1}}{m_{0}}\right) E(t) \\
& +\int_{\Omega}\left[\left(n-\left(\frac{n}{2}-\theta\right) \beta\right) a(x)+m \cdot \nabla a\right] F(u) d x .
\end{aligned}
$$


At this point, we choose

$$
\varepsilon=\varepsilon_{1}<\min \left\{\frac{m_{0}}{4}, \frac{(1-\theta)}{2\left(c_{0}+\left(\frac{n}{2}-\theta\right)^{2} B_{*}\right)}\right\} .
$$

Once $\varepsilon=\varepsilon_{1}$ is fixed (hence $c_{\varepsilon}$ and $c_{\varepsilon_{1}}$ are also fixed), we pick $N$ large satisfying (3.12) and

$$
N>\max \left\{\frac{\max _{\Gamma_{1}}|m \cdot v|+16 \tau^{2} c_{\varepsilon_{1}}}{\tau}, c_{\varepsilon}\right\}
$$

at the same time. Then, from the properties of $k(t)$ by (3.1) and noting that $|g \diamond \phi(t)|^{2} \leq \int_{0}^{t}|g(s)| d s(|g| \circ \phi)(t)$ by (2.4), we see that

$$
\begin{aligned}
L^{\prime}(t) \leq & -\theta\left\|u_{t}\right\|_{2}^{2}-\frac{1-\theta}{2} M\left(\|\nabla u\|_{2}^{2}\right)\|\nabla u\|_{2}^{2} \\
& -k(0) \int_{\Gamma_{1}} k^{\prime} \circ u d \Gamma+c_{4} k^{2}(t) \int_{\Gamma_{1}}\left|u_{0}\right|^{2} d \Gamma \\
& +8 \tau^{2} c_{\varepsilon_{1}} k^{2}(t) \int_{\Gamma_{1}}|u|^{2} d \Gamma+\beta_{1} \gamma(0)\left(N c_{3}+\frac{2 c_{1}}{m_{0}}\right) E(t) \\
& +\int_{\Omega}\left[\left(n-\left(\frac{n}{2}-\theta\right) \beta\right) a(x)+m \cdot \nabla a\right] F(u) d x
\end{aligned}
$$

Utilizing the inequality $M(\lambda) \lambda \geq \widehat{M}(\lambda)$ by (1.12) and the definition of $E(t)$ by (3.5), we obtain

$$
\begin{aligned}
L^{\prime}(t) \leq & -\left(\alpha-\beta_{1} \gamma(0)\left(N c_{3}+\frac{2 c_{1}}{m_{0}}\right)\right) E(t)+\left(\frac{\tau \alpha}{2} k(t)+8 \tau^{2} c_{\varepsilon_{1}} k^{2}(t)\right) \int_{\Gamma_{1}}|u|^{2} d \Gamma \\
& -\left(\frac{\tau \alpha}{2}+k(0)\right) \int_{\Gamma_{1}} k^{\prime} \circ u d \Gamma+c_{4} k^{2}(t) \int_{\Gamma_{1}}\left|u_{0}\right|^{2} d \Gamma \\
& +\int_{\Omega}\left[\left(n+\alpha-\left(\frac{n}{2}-\theta\right) \beta\right) a(x)+m \cdot \nabla a\right] F(u) d x,
\end{aligned}
$$

which together with (3.19) and (3.10) infers that

$$
\begin{aligned}
L^{\prime}(t) \leq & -\left(\alpha-\beta_{1} \gamma(0)\left(N c_{3}+\frac{2 c_{1}}{m_{0}}\right)\right) E(t)+\frac{2 B_{*}}{m_{0}}\left(\frac{\tau \alpha}{2} k(t)+8 \tau^{2} c_{\varepsilon_{1}} k^{2}(t)\right) E(t) \\
& -\left(\frac{\tau \alpha}{2}+k(0)\right) \int_{\Gamma_{1}} k^{\prime} \circ u d \Gamma+c_{4} k^{2}(t) \int_{\Gamma_{1}}\left|u_{0}\right|^{2} d \Gamma \\
& +\int_{\Omega}\left[\left(n+\alpha-\left(\frac{n}{2}-\theta\right) \beta\right) a(x)+m \cdot \nabla a\right] F(u) d x
\end{aligned}
$$

where $\alpha=\min \{2 \theta, 1-\theta\}$. Besides, we note that there exists $t_{0}$ large enough satisfying

$$
k(t) \leq \frac{m_{0}}{2 B_{*}} \min \left\{\sqrt{\frac{\alpha}{64 \tau^{2} c_{\varepsilon_{2}}}}, \frac{1}{4 \tau}\right\} \quad \text { for } t \geq t_{0},
$$

because of $\lim _{t \rightarrow \infty} k(t)=0$ by (3.13). Therefore, taking $\beta_{1}$ small enough such that

$$
0<\beta_{1}<\frac{\alpha}{4 \gamma(0)\left(N c_{3}+\frac{2 c_{1}}{m_{0}}\right)}
$$


then,

$$
\begin{aligned}
L^{\prime}(t) \leq & -\frac{\alpha}{2} E(t)+c_{4} k^{2}(t) \int_{\Gamma_{1}}\left|u_{0}\right|^{2} d \Gamma-c_{5} \int_{\Gamma_{1}} k^{\prime} \circ u d \Gamma \\
& +\int_{\Omega}\left[\left(n+\alpha-\left(\frac{n}{2}-\theta\right) \beta\right) a(x)+m \cdot \nabla a\right] F(u) d x
\end{aligned}
$$

for all $t \geq t_{0}$, where $c_{i}$ are positive constants, $i=4,5$. This completes the proof.

Theorem 3.5. Given that $\left(u_{0}, u_{1}\right) \in\left(H^{2}(\Omega) \cap V\right)^{2}$, assume that (A1)-(A4), (3.1)-(3.3) and (3.13)hold, with $\beta_{1}$ (given by (A4)) small enough. Assume further that

$$
\left(n+\alpha-\left(\frac{n}{2}-\theta\right) \beta\right) a(x)+m \cdot \nabla a<0, \quad \forall x \in \Omega .
$$

Then, for some $t_{0}$ large enough, we have, $\forall t \geq t_{0}$,

$$
E(t) \leq c E\left(t_{0}\right) e^{-a_{1} \int_{0}^{t} \gamma(s) d s} \quad \text { if } u_{0}=0 \text { on } \Gamma_{1},
$$

otherwise (if $u_{0} \neq 0$ on $\Gamma_{1}$ ),

$$
E(t) \leq c\left\{E\left(t_{0}\right)+\left(\int_{\Gamma_{1}}\left|u_{0}\right|^{2} d \Gamma\right) \int_{t_{0}}^{t} k^{2}(s) e^{a_{1} \int_{t_{0}}^{s} \gamma(\zeta) d \zeta} d s\right\} e^{-a_{1} \int_{0}^{t} \gamma(s) d s},
$$

where $a_{1}$ is a fixed positive constant and cis a generic positive constant.

Proof. Multiplying (3.25) by $\gamma(t)$ and exploiting (3.26), (3.1) and (3.7), we derive that

$$
\begin{aligned}
\gamma(t) L^{\prime}(t) & \leq-\frac{\alpha}{2} \gamma(t) E(t)+c_{4} k^{2}(t) \gamma(t) \int_{\Gamma_{1}}\left|u_{0}\right|^{2} d \Gamma-c_{5} \gamma(t) \int_{\Gamma_{1}} k^{\prime} \circ u d \Gamma \\
& \leq-\frac{\alpha}{2} \gamma(t) E(t)+c_{4} k^{2}(t) \gamma(t) \int_{\Gamma_{1}}\left|u_{0}\right|^{2} d \Gamma+c_{5} \int_{\Gamma_{1}} k^{\prime \prime} \circ u d \Gamma \\
& \leq-\frac{\alpha}{2} \gamma(t) E(t)+c_{6} k^{2}(t) \int_{\Gamma_{1}}\left|u_{0}\right|^{2} d \Gamma-c_{7} E^{\prime}(t) \\
& -c_{7} l(t) \int_{\Omega} h(\nabla u) u_{t} d x
\end{aligned}
$$

where $c_{6}=c_{4} \gamma(0)+c_{5}$ and $c_{7}=\frac{2 c_{5}}{\tau}$. Employing (3.21) again, (3.29) becomes

$$
F_{1}^{\prime}(t)-\gamma^{\prime}(t) L(t) \leq-\gamma(t)\left(\frac{\alpha}{2}-\beta_{1} c_{7} c_{3}\right) E(t)+c_{6} k^{2}(t) \int_{\Gamma_{1}}\left|u_{0}\right|^{2} d \Gamma,
$$

where

$$
F_{1}(t)=\gamma(t) L(t)+c_{7} E(t),
$$

which is equivalent to $E(t)$ due to Lemma 3.3 and $\gamma(t)$ is nonincreasing by (3.2). In addition to (3.24), we further require

$$
0<\beta_{1}<\frac{\alpha}{8 c_{7} c_{3}},
$$

then, we have

$$
F_{1}^{\prime}(t) \leq-a_{1} \gamma(t) F_{1}(t)+c_{6} k^{2}(t) \int_{\Gamma_{1}}\left|u_{0}\right|^{2} d \Gamma, \forall t \geq t_{0},
$$


where $a_{1}$ is a positive constant.

Case I: If $u_{0}=0$ on $\Gamma_{1}$, then (3.30) reduces to

$$
F_{1}^{\prime}(t) \leq-a_{1} \gamma(t) F_{1}(t), \quad \forall t \geq t_{0} .
$$

Integrating the above inequality over $\left(t_{0}, t\right)$ to get

$$
F_{1}(t) \leq F_{1}\left(t_{0}\right) e^{-a_{1} \int_{t_{0}}^{t} \gamma(s) d s}, \quad \forall t \geq t_{0} .
$$

Then, using the fact $F_{1}(t)$ is equivalent to $E(t)$, we obtain, for some positive constant $c$,

$$
\begin{aligned}
E(t) & \leq c E\left(t_{0}\right) e^{-a_{1} \int_{t_{0}}^{t} \gamma(s) d s} \\
& =c E\left(t_{0}\right) e^{a_{1} \int_{0}^{t_{0}} \gamma(s) d s} e^{-a_{1} \int_{0}^{t} \gamma(s) d s}, \quad \forall t \geq t_{0} .
\end{aligned}
$$

Thus, (3.27) is proved.

Case II: If $u_{0} \neq 0$ on $\Gamma_{1}$, then (3.30) gives

$$
F_{1}^{\prime}(t) \leq-a_{1} \gamma(t) F_{1}(t)+c_{8} k^{2}(t), \quad \forall t \geq t_{0}
$$

where $c_{8}=c_{6} \int_{\Gamma_{1}}\left|u_{0}\right|^{2} d \Gamma$. Direct computations give

$$
\left(e^{a_{1} \int_{t_{0}}^{t} \gamma(s) d s} F_{1}(t)\right)^{\prime} \leq c_{8} k^{2}(t) e^{a_{1} \int_{t_{0}}^{t} \gamma(s) d s} .
$$

An integration over $\left(t_{0}, t\right)$ yields

$$
F_{1}(t) \leq\left(F_{1}\left(t_{0}\right)+c_{8} \int_{t_{0}}^{t} k^{2}(s) e^{a_{1} \int_{t_{0}}^{s} \gamma(\zeta) d \zeta} d s\right) e^{-a_{1} \int_{t_{0}}^{t} \gamma(s) d s}, \quad \forall t \geq t_{0} .
$$

Again using the fact $F_{1}(t)$ is equivalent to $E(t)$, we obtain, for some positive constant $c$,

$$
E(t) \leq c\left\{E\left(t_{0}\right)+\left(\int_{\Gamma_{1}}\left|u_{0}\right|^{2} d \Gamma\right) \int_{t_{0}}^{t} k^{2}(s) e^{a_{1} \int_{t_{0}}^{s} \gamma(\xi) d \zeta} d s\right\} e^{a_{1} \int_{0}^{t_{0}} \gamma(s) d s} e^{-a_{1} \int_{0}^{t} \gamma(s) d s}, \quad \forall t \geq t_{0} .
$$

This completes the proof of Theorem 3.5.

\section{Conclusion and suggestions}

Santos et al. [22] considered problem (1.1)-(1.4) with $a=1$ and without a function of the gradient term. They showed the solution decays exponentially (or polynomially) to zero provided the kernel decays exponentially (or polynomially) to zero. Recently, Messaoudi and Soufyane in 2010 [17] considered a semi-linear wave equation, in a bounded domain, where the memory-type damping is acting on the boundary. They established a general decay result, from which the usual exponential and polynomial decay rate are only special cases. Motivated by this, we intended to investigate the decay properties of problem (1.1)-(1.4) using the work of Messaaoudi and Soufyane [17]. Since stability of problems with the nonlinear term $h(\nabla u)$ requires a careful treatment, it is interesting to investigate whether we still have the similar general decay result as that of [16] in the presence of a function of the gradient term. This is our motivation to consider problem (1.1)-(1.4). And, this problem is not considered before. 
By adopting and modifying the method proposed by Messaoudi and Soufyane in 2010 [17], we establish a general decay result, from which the usual exponential and polynomial decay rate are only special cases. Further, our result allows certain kernels which are not necessarily of exponential or polynomial decay. In this way, we improved the results of Santos et al. [22], in which they considered problem (1.1)-(1.4) with $a=$ 1 and in the absence of $l(t) h(\nabla u)$. Moreover, we note that our result also holds for problem (1.1)-(1.4) with $a=1$ and $l(t)=0$ and without imposing strong damping term, thus our result improves the one of Bae et al. [27]. More precisely, the estimate (3.27) and (3.28) generalizes the exponential and polynomial decay result given in [22,27]. Indeed, we obtain exponential decay for $\gamma(t)=c$ and polynomial decay for $\gamma(t)$ $=c(1+t)^{-1}$, where $c$ is a positive constant. Additionally, as in [17], our result allows kernels which satisfy $k^{\prime \prime}(t) \geq c\left(-k^{\prime}\right)^{1+q}$, for $0<q<1$ instead of the usual assumption $0<q<\frac{1}{2}$. It suffices to take, for example, $k(t)=(1+t)^{-\lambda}$, for $\lambda>0$. Direct computations yield

$$
k^{\prime \prime}(t)=c\left(-k^{\prime}(t)\right)^{1+\frac{1}{1+\lambda}} .
$$

It is clear that $0<\frac{1}{1+\lambda}<1$, for $\lambda>0$.

Though we consider the conditions on the term involving the gradient are too restrictive and we combine some known ideas to obtain our result, our findings extend those decay results in [22,27] and these findings are interesting to those with closely concerns. For future work, we will consider not necessarily decreasing kernels and relax the condition of $h(\nabla u)$.

\section{Acknowledgements}

The author would like to thank the anonymous referees for their valuable and constructive suggestions which improve this work.

\section{Competing interests}

The author declare that they have no competing interests.

Received: 30 July 2011 Accepted: 23 December 2011 Published: 23 December 2011

References

1. Kirchhoff, G: Vorlesungen über Mechanik. Leipzig Teubner. (1883)

2. Biler, P: Remark on the decay for damped string and beam equations. Nonlinear Anal TMA. 9, 839-842 (1984)

3. Brito, EH: Nonlinear initial boundary value problems. Nonlinear Anal TMA. 11, 125-137 (1987). doi:10.1016/0362-546X(87) 90031-9

4. Ikerata, R: On the existence of global solutions for some nonlinear hyperbolic equations with Neumann conditions. TRU Math. 24, 1-17 (1988)

5. Ikerata, R: A note on the global solvability of solutions to some nonlinear wave equations with dissipative terms. Differential Integral Equations. 8, 607-616 (1995)

6. Matos, MP, Pereira, DC: On a hyperbolic equation with strong damping. Funkcial Ekvac. 34, 303-311 (1991)

7. Matsuyama, T, Ikerata, R: On global solutions and energy decay for the wave equations of Kirchhoff type with nonlinear damping terms. J Math Anal Appl. 204, 729-753 (1996). doi:10.1006/jmaa.1996.0464

8. Nishihara, K, Yamada, Y: On global solutions of some degenerate quasilinear hyperbolic equations with dissipative terms. Funkcial Ekvac. 33, 151-159 (1990)

9. Nishihara, K: Exponentially decay of solutions of some quasilinear hyperbolic equations with linear damping. Nonlinear Anal TMA. 8, 623-636 (1984). doi:10.1016/0362-546X(84)90007-5

10. Ono, K: On global existence, asymptotic stability and blowing up of solutions for some degenerate nonlinear wave equations of Kirchhoff type with a strong dissipation. Math Methods Appl Sci. 20, 151-177 (1997). doi:10.1002/(SICI) 1099-1476(19970125)20:23.0.CO;2-0

11. Ono, K: On global solutions and blow-up solutions of nonlinear Kirchhoff strings with nonlinear dissipation. J Math Anal Appl. 216, 321-342 (1997). doi:10.1006/jmaa.1997.5697

12. Wu, ST, Tsai, LY: Blow-up of solutions for some nonlinear wave equations of Kirchhoff type with some dissipation. Nonlinear Anal TMA. 65, 243-264 (2006). doi:10.1016/j.na.2004.11.023

13. Yamada, Y: On some quasilinear wave equations with dissipative terms. Nagoya Math J. 87, 17-39 (1982) 
14. Cavalcanti, MM, Domingos Cavalcanti, VN, Prates, JS, Soriano, JA: Existence and uniform decay of solutions of a degenerate equation with nonlinear boundary memory source term. Nonlinear Anal TMA. 38, 281-294 (1999). doi:10.1016/50362-546X(98)00195-3

15. Cavalcanti, MM, Guesmia, A: General decay rates of solutions to a nonlinear wave equation with boundary condition of memory type. Differential Integral Equations. 18, 583-600 (2005)

16. Messaoudi, SA, Soufyane, A: Boundary stabilization of solutions of a nonlinear system of Timoshenko system. Nonlinear Anal TMA. 67, 2107-2121 (2007). doi:10.1016/j.na.2006.08.039

17. Messaoudi, SA, Soufyane, A: General decay of solutions of a wave equation with a boundary control of memory type. Nonlinear Anal Real World Appl. 11, 2896-2904 (2010). doi:10.1016/j.nonrwa.2009.10.013

18. Cavalcanti, MM, Domingos Cavalcanti, VN, Prates, JS, Soriano, JA: Existence and uniform decay rates for viscoelastic problems with nonlinear boundary damping. Differential Integral Equations. 14, 85-116 (2001)

19. Cavalcanti, MM, Domingos Cavalcanti, VN, Santos, ML: Existence and uniform decay rates of solutions to a degenerate system with memory conditions at the boundary. Appl Math Comput. 150, 439-465 (2004). doi:10.1016/50096-3003(03) 00284-4

20. Jiang, S, Muñoz Rivera, JE: A global existence for the Dirichlet problems in nonlinear n-dimensional viscoelasticity. Differential Integral Equations. 9, 791-810 (1996)

21. Santos, ML: Asymptotic behavior of solutions to wave equations with a memory condition at the boundary. Electronic J Differential Equations. 73, 1-11 (2001)

22. Santos, ML, Ferreira, J, Pereira, DC, Raposo, CA: Global existence and stability for wave equation of Kirchhoff type with memory condition at the boundary. Nonlinear Anal TMA. 54, 959-976 (2003). doi:10.1016/50362-546X(03)00121-4

23. Park, JY, Bae, JJ, Hyo Jung, IL: Uniform decay of solution for wave equation of Kirchhoff type with nonlinear boundary damping and memory term. Nonlinear Anal TMA. 50, 871-884 (2002). doi:10.1016/50362-546X(01)00781-7

24. Aassila, M, Cavalcanti, MM, Domingos Cavalcanti, VN: Existence and uniform decay of the wave equation with nonlinear boundary damping and boundary memory source term. Calc Var. 15, 155-180 (2002). doi:10.1007/s005260100096

25. Guesmia, A: A new approach of stabilization of nondissipative distributed systems. SIAM J Control Optim. 42, 24-52 (2003). doi:10.1137/S0363012901394978

26. Park, JY, Ha, TG: Energy decay for nondissipative distributed systems with boundary damping and source term. Nonlinear Anal TMA. 70, 2416-2434 (2009). doi:10.1016/j.na.2008.03.026

27. Bae, JJ, Yoon, SB: On uniform decay of wave equation of carrier model subject to memory condition at the boundary. J Korean Math Soc. 44, 1013-1024 (2007). doi:10.4134/JKMS.2007.44.4.1013

28. Santos, ML, Junior, F: A boundary condition with memory for Kirchhoff plate equations. Appl Math Comput. 148, 475-496 (2004). doi:10.1016/S0096-3003(02)00915-3

doi:10.1186/1687-2770-2011-55

Cite this article as: Wu: General decay for a wave equation of Kirchhoff type with a boundary control of memory type. Boundary Value Problems 2011 2011:55.

\section{Submit your manuscript to a SpringerOpen ${ }^{\odot}$ journal and benefit from:}

- Convenient online submission

- Rigorous peer review

- Immediate publication on acceptance

- Open access: articles freely available online

- High visibility within the field

- Retaining the copyright to your article

Submit your next manuscript at $\gg$ springeropen.com 